Reports From the Field

\title{
COVID-19 Screening and Testing Among Patients With Neurologic Dysfunction: The Neuro-COVID-19 Time-out Process and Checklist
}

\author{
Prashant A. Natteru, MD, Hartmut Uschmann, MD, and Christa O'Hana S. Nobleza, MD, MSCl
}

\section{ABSTRACT}

Objective: To test a coronavirus disease 2019 (COVID19) screening tool to identify patients who qualify for testing among patients with neurologic dysfunction who are unable to answer the usual screening questions, which could help to prevent unprotected exposure of patients and health care workers to COVID-19.

Methods: The Neuro-COVID-19 Time-out Process and Checklist (NCOT-PC) was implemented at our institution for 1 week as a quality improvement project to improve the pathway for COVID-19 screening and testing among patients with neurologic dysfunction.

Results: A total of 14 new patients were admitted into the neuroscience intensive care unit (NSICU) service during the pilot period. The NCOT-PC was utilized on 9 (64\%) patients with neurologic dysfunction; 7 of these patients were found to have a likelihood of requiring testing based on the NCOT-PC and were subsequently screened for COVID-19 testing by contacting the institution's COVID-19 testing hotline (Med-Com). All these patients were subsequently transitioned into person-under-investigation status based on the determination from Med-Com. The NSICU staff involved were able to utilize NCOT-PC without issues. The NCOT-PC was immediately adopted into the NSICU process.

Conclusion: Use of the NCOT-PC tool was found to be feasible and improved the screening methodology of patients with neurologic dysfunction.

Keywords: coronavirus; health care planning; quality improvement; patient safety; medical decision-making; neuroscience intensive care unit. he coronavirus disease 2019 (COVID-19) pan-
demic has altered various standard emergent
care pathways. Current recommendations regarding COVID-19 screening for testing involve asking patients about their symptoms, including fever, cough, chest pain, and dyspnea. ${ }^{1}$ This standard screening method poses a problem when caring for patients with neurologic dysfunction. COVID-19 patients may present with conditions that affect their ability to answer questions, such as stroke, encephalitis, neuromuscular disorders, or headache, and that may preclude the use of standard screening for testing. ${ }^{2}$ Patients with acute neurologic dysfunction who cannot undergo standard screening may leave the emergency department (ED) and transition into the neuroscience intensive care unit (NSICU) or any intensive care unit (ICU) without a reliable COVID-19 screening test.

The Protected Code Stroke pathway offers protection in the emergent setting for patients with stroke when their COVID-19 status is unknown. ${ }^{3} \mathrm{~A}$ similar process has been applied at our institution for emergent management of patients with cerebrovascular disease (stroke, intracerebral hemorrhage, and subarachnoid hemorrhage). However, the process from the ED after designating "difficult to screen" patients as persons under investigation (PUI) is unclear. The Centers for Disease Control and Prevention (CDC) has delineated the priorities for testing, with not all declared PUls requiring testing. ${ }^{4}$ This poses a great challenge, because patients designated as PUIs require the same management as a COVID-19-positive patient, with negative-pressure isolation rooms as well as use of protective personal equipment (PPE), which

From the University of Mississippi Medical Center, Department of Neurology, Division of Neuroscience Intensive Care, Jackson, MS. 


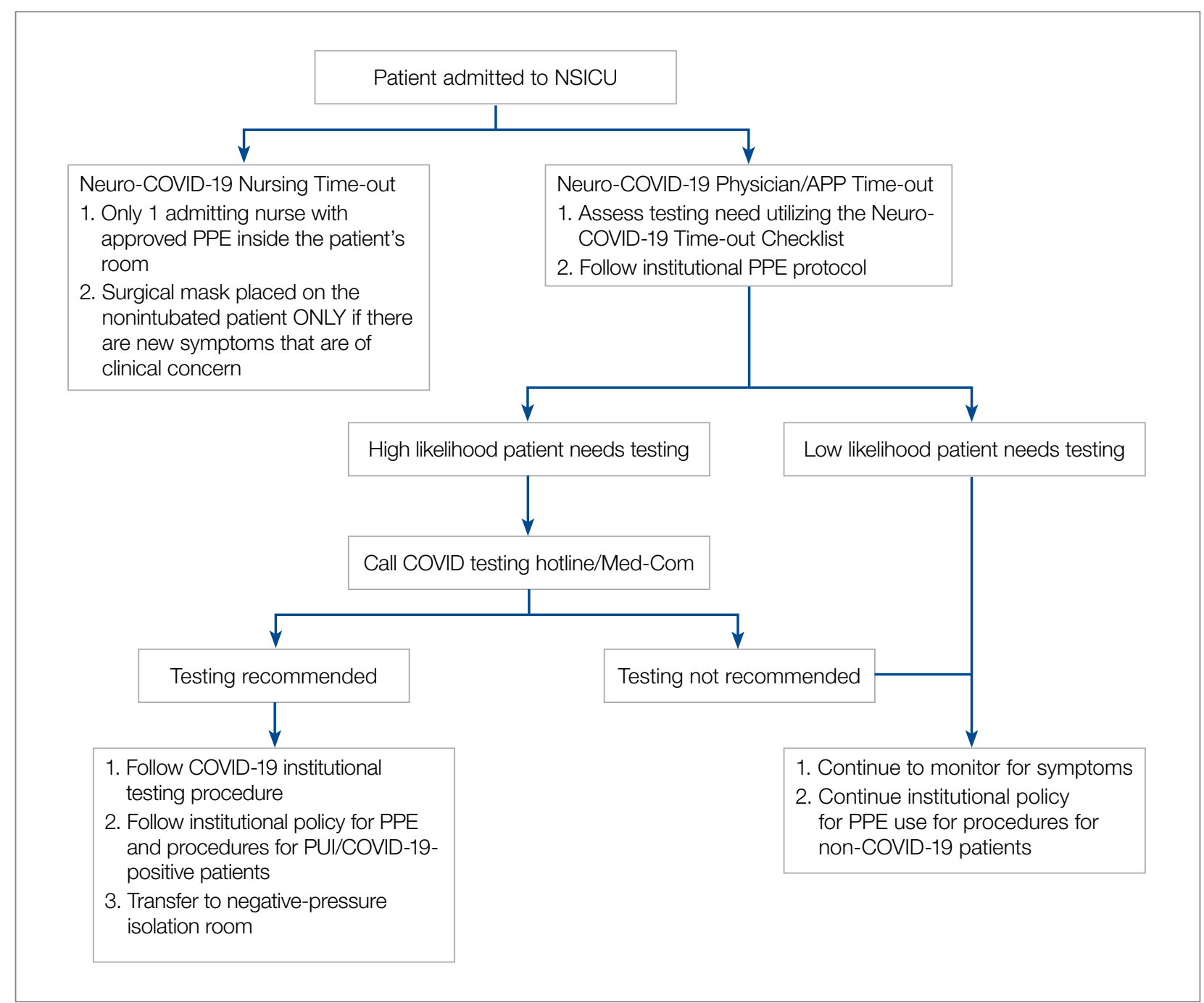

Figure 1. Neuro-COVID-19 Time-out Process algorithm in the neuroscience intensive care unit (NSICU). PPE, personal protective equipment; PUI, persons under investigation.

may not be readily available. It was also recognized that, because the ED staff can be overwhelmed by COVID-19 patients, there may not be enough time to perform detailed screening of patients with neurologic dysfunction and that "reverse masking" may not be done consistently for nonintubated patients. This may place patients and health care workers at risk of unprotected exposure.

Recognizing these challenges, we created a NeuroCOVID-19 Time-out Process and Checklist (NCOT-PC) as a quality improvement project. The aim of this project was to improve and standardize the current process of identifying patients with neurologic dysfunction who require COVID-19 testing to decrease the risk of unprotected exposure of patients and health care workers.

\section{Methods}

\section{Patients and Definitions}

This quality improvement project was undertaken at the University of Mississippi Medical Center NSICU. Because this was a quality improvement project, an Institutional Review Board exemption was granted.

The NCOT-PC was utilized in consecutive patients with neurologic dysfunction admitted to the NSICU during a period of 1 week. "Neurologic dysfunction" 
Neuro-COVID-19 Time-out Checklist for Clinicians

Call to Med-Com/COVID hotline if:

Neurologic issue + any of A or B + C or D; C + D; or D + strong clinical suspicion

\begin{tabular}{|l|l|}
\hline A. & Assess risk factors in terms of location: \\
$\square$ & Any international travel \\
$\square$ & Domestic travel from endemic areas (based on CDC guidance) \\
$\square$ & State location with high exposure (see state health department) \\
\hline B. & Assess risk factors in terms of history and exposure: \\
$\square$ & Exposure to COVID (+) with or without PPE (HCW, patients) \\
$\square$ & Fever \\
$\square$ & Upper respiratory symptoms (dry cough, sputum production, sore throat) \\
$\square$ & Runny nose and nasal congestion \\
$\square$ & Shortness of breath \\
$\square$ & Chest tightness \\
$\square$ & Headache \\
$\square$ & Gastrointestinal symptoms (diarrhea, vomiting) \\
$\square$ & Loss of sense of smell \\
$\square$ & Loss of sense of taste \\
$\square$ & Fatigue, myalgias, malaise, and other flu-like symptoms \\
$\square$ & Assess risk factors in terms of clinical examination: \\
\hline C. & Check ER and OSH temperature, determine if febrile \\
$\square$ & Check oxygen saturation and requirement \\
$\square$ & Any new oxygen requirement \\
$\square$ & Assess risk factors in terms of imaging findings: \\
\hline D. & CT chest: ground-glass opacity, bilateral patchy shadowing (from CTA head and neck), CT C-spine or CT CAP \\
$\square$ & Chest x-ray: unexplained opacities (bilateral/unilateral) \\
$\square$ &
\end{tabular}

Figure 2. Neuro-COVID-19 Time-out Checklist for assessing the likelihood (high versus low) COVID-19 testing is needed in patients with neurologic dysfunction. CDC, Centers for Disease Control and Prevention; CT, computed tomography; CTA, CT angiogram; CT CAP, CT chest/abdomen/pelvis; ER, emergency room; HCW, health care worker; OSH, outside hospital; PPE, personal protective equipment.

encompasses any neurologic illness affecting the mental status and/or level of alertness, subsequently precluding the ability to reliably screen the patient utilizing standard COVID-19 screening. "Med-Com" at our institution is the equivalent of the national COVID-19 testing hotline, where our institution's infectious diseases experts screen calls for testing and determine whether testing is warranted. "Unprotected exposure" means exposure to COVID-19 without adequate and appropriate PPE.

\section{Quality Improvement Process}

As more PUls were being admitted to the institution, we used the Plan-Do-Study-Act method for process improvements in the NSICU. ${ }^{5} \mathrm{NSICU}$ stakeholders, including attendings, the nurse manager, and nurse practitioners (NPs), developed an algorithm to facilitate the coordination of the NSICU staff in screening patients to identify those with a high likelihood of needing COVID19 testing upon arrival in the NSICU (Figure 1). Once the NCOT-PC was finalized, NSICU stakeholders were educated regarding the use of this screening tool.

The checklist clinicians review when screening patients is shown in Figure 2. The risk factors comprising the checklist include patient history and clinical and radiographic characteristics that have been shown to be relevant for identifying patients with COVID-19.6,7 The imaging criteria utilize imaging that is part of the standard of care for NSICU patients. For example, computed tomography 


\begin{tabular}{|c|c|c|c|}
\hline $\begin{array}{l}\text { Patient } \\
\text { Number }\end{array}$ & Primary Admitting Diagnosis & $\begin{array}{l}\text { Neurologic Deficit/Characteristic } \\
\text { Precluding Screening }\end{array}$ & Neuro-COVID-19 Time-out Category \\
\hline 1 & $\begin{array}{l}\text { Aneurysmal subarachnoid } \\
\text { hemorrhage }\end{array}$ & Abulia & $\begin{array}{l}\text { Increased oxygen requirement + CTA } \\
\text { neck: ground-glass opacities }\end{array}$ \\
\hline 2 & Acute ischemic stroke & Aphasia & $\begin{array}{l}\text { New oxygen requirement + CTA neck: } \\
\text { ground-glass opacities }\end{array}$ \\
\hline 3 & Subdural hematoma & Mute & New oxygen requirement + cough \\
\hline 4 & Acute ischemic stroke & Confused & CTA neck: tree-in-bud findings \\
\hline 5 & Status epilepticus & Intubated & CT chest: right lower lobe consolidation \\
\hline 6 & $\begin{array}{l}\text { Aneurysmal subarachnoid } \\
\text { hemorrhage }\end{array}$ & Intubated & Travel/location history + cough \\
\hline 7 & Thalamic tumor & Intubated & Location history + cough \\
\hline
\end{tabular}

angiogram of the head and neck performed as part of the acute stroke protocol captures the upper part of the chest. These images are utilized for their incidental findings, such as apical ground-glass opacities and tree-inbud formation. The risk factors applicable to the patient determine whether the clinician will call Med-Com for testing approval. Institutional COVID-19 processes were then followed accordingly. ${ }^{8}$ The decision from Med-Com was considered final, and no deviation from institutional policies was allowed.

NCOT-PC was utilized for consecutive days for 1 week before re-evaluation of its feasibility and adaptability.

\section{Data Collection and Analysis}

Consecutive patients with neurologic dysfunction admitted into the NSICU were assigned nonlinkable patient numbers. No identifiers were collected for the purpose of this project. The primary diagnosis for admission, the neurologic dysfunction that precluded standard screening, and checklist components that the patient fulfilled were collected.

To assess the tool's feasibility, feedback regarding the ease of use of the NCOT-PC was gathered from the nurses, NPs, charge nurses, fellows, and other attendings. To assess the utility of the NCOT-PC in identifying patients who will be approved for COVID-19 testing, we calculated the proportion of patients who were deemed to have a high likelihood of testing and the proportion of patients who were approved for testing. Descriptive statistics were used, as applicable for the project, to summarize the utility of the NCOT-PC.

\section{Results}

We found that the NCOT-PC can be easily used by clinicians. The NSICU staff did not communicate any implementation issues, and since the NCOT-PC was implemented, no problems have been identified.

During the pilot period of the NCOT-PC, 14 new patients were admitted to the NSICU service. Nine (64\%) of these had neurologic dysfunction, and the NCOT-PC was used to determine whether Med-Com should be called based on the patients' likelihood (high vs low) of needing a COVID-19 test. Of those patients with neurologic dysfunction, 7 (78\%) were deemed to have a high likelihood of needing a COVID-19 test based on the NCOT-PC. Med-Com was contacted regarding these patients, and all were deemed to require the COVID-19 test by Med-Com and were transitioned into PUI status per institutional policy (Table).

\section{Discussion}

The NCOT-PC project improved and standardized the process of identifying and screening patients with neurologic dysfunction for COVID-19 testing. The screening tool is feasible to use, and it decreased inadvertent unprotected exposure of patients and health care workers. 
The NCOT-PC was easy to administer. Educating the staff regarding the new process took only a few minutes and involved a meeting with the nurse manager, NPs, fellows, residents, and attendings. We found that this process works well in tandem with the standard institutional processes in place in terms of Protected Code Stroke pathway, PUI isolation, PPE use, and Med-Com screening for COVID-19 testing. Med-Com was called only if the patient fulfilled the checklist criteria. In addition, no extra cost was attributed to implementing the NCOT-PC, since we utilized imaging that was already done as part of the standard of care for patients with neurologic dysfunction.

The standardization of the process of screening for COVID-19 testing among patients with neurologic dysfunction improved patient selection. Before the NCOT-PC, there was no consistency in terms of who should get tested and the reason for testing patients with neurologic dysfunction. Patients can pass through the ED and arrive in the NSICU with an unclear screening status, which may cause inadvertent patient and health care worker exposure to COVID-19. With the NCOT-PC, we have avoided instances of inadvertent staff or patient exposure in the NSICU.

The NCOT-PC was adopted into the NSICU process after the first week it was piloted. Beyond the NSICU, the application of the NCOT-PC can be extended to any patient presentation that precludes standard screening, such as ED and interhospital transfers for stroke codes, trauma codes, code blue, or myocardial infarction codes. In our department, as we started the process of PCS for stroke codes, we included NCOT-PC for stroke patients with neurologic dysfunction.

The results of our initiative are largely limited by the decision-making process of Med-Com when patients are called in for testing. At the time of our project, there were no specific criteria used for patients with altered mental status, except for the standard screening methods, and it was through clinician-to-clinician discussion that testing decisions were made. Another limitation is the short period of time that the NCOT-PC was applied before adoption.
In summary, the NCOT-PC tool improved the screening process for COVID-19 testing in patients with neurologic dysfunction admitted to the NSICU. It was feasible and prevented unprotected staff and patient exposure to COVID-19. The NCOT-PC functionality was compatible with institutional COVID-19 policies in place, which contributed to its overall sustainability.

The Standards for Quality Improvement Reporting Excellence (SQUIRE 2.0) were utilized in preparing this manuscript. $^{9}$

Acknowledgment: The authors thank the University of Mississippi Medical Center NSICU staff for their input with implementation of the NCOT-PC.

Corresponding author: Prashant A. Natteru, MD, University of Mississippi Medical Center, Department of Neurology, 2500 North State St., Jackson, MS 39216; pnatteru@umc.edu.

Financial disclosures: None.

doi:10.12788/jcom.0021

\section{References}

1. Coronavirus disease 2019 (COVID-19) Symptoms. www.cdc. gov/coronavirus/2019-ncov/symptoms-testing/symptoms.html. Accessed April 9, 2020.

2. Mao $L$, Jin $H$, Wang $M$, et al. Neurologic manifestations of hospitalized patients with coronavirus disease 2019 in Wuhan, China. JAMA Neurol. 2020;77:1-9.

3. Khosravani H, Rajendram P, Notario L, et al. Protected code stroke: hyperacute stroke management during the coronavirus disease 2019. (COVID-19) pandemic. Stroke. 2020;51:1891-1895.

4. Coronavirus disease 2019 (COVID-19) evaluation and testing. www.cdc.gov/coronavirus/2019-nCoV/hcp/clinical-criteria.html. Accessed April 9, 2020.

5. Plan-Do-Study-Act Worksheet. Institute for Healthcare Improvement website. www.ihi.org/resources/Pages/Tools/ PlanDoStudyActWorksheet.aspx. Accessed March 31,2020.

6. Li YC, Bai WZ, Hashikawa T. The neuroinvasive potential of SARSCoV2 may play a role in the respiratory failure of COVID-19 patients. J Med Virol. 2020;10.1002/jmv.25728.

7. Rodriguez-Morales AJ, Cardona-Ospina JA, Gutiérrez-Ocampo E, et al. Clinical, laboratory and imaging features of COVID-19: A systematic review and meta-analysis. Travel Med Infect Dis. 2020;101623.

8. UMMC's COVID-19 Clinical Processes. www.umc.edu/CoronaVirus/ Mississippi-Health-Care-Professionals/Clinical-Resources/ClinicalResources.html. Accessed April 9, 2020.

9. SQUIRE 2.0 (Standards for QUality Improvement Reporting Excellence): Revised Publication Guidelines from a Detailed Consensus Process. The EQUATOR Network. www.equator-network.org/reporting-guidelines/squire/. Accessed May 12, 2020. 